\title{
EVALUACIÓN DE LA ACTIVIDAD ANTIVIRULENCIA DE ACEITES ESENCIALES CONTRA Pseudomonas aeruginosa
}

\author{
ASSESSMENT OF ANTIVIRULENCE ACTIVITY OF ESSENTIAL OILS \\ AGAINST Pseudomonas aeruginosa
}

\section{Araceli Blanco-Hernández¹, Rodolfo García-Contreras³ ${ }^{3}$ Rosa E. Sánchez-Fernández', Mariana Palma-Tenango ${ }^{1,4}$, Juan Cibrián-Tovar ${ }^{1}$, Naybi Muñoz-Cazares ${ }^{1}$, R. Marcos Soto-Hernández ${ }^{1}$, Daniel Padilla-Chacon ${ }^{1,2}$, F. Javier Prado-Galbarro ${ }^{5,6}$ e Israel Castillo-Juárez ${ }^{1,2 *}$}

\begin{abstract}
${ }^{1}$ Colegio de Postgraduados, Campus Montecillo, Posgrado de Botánica, Montecillo, Texcoco, Estado de México, México. ${ }^{2}$ CONACYT-Colegio de Postgraduados, Campus Montecillo, Posgrado de Botánica, Montecillo, Texcoco, Estado de México, México. ${ }^{3}$ Universidad Nacional Autónoma de México, Facultad de Medicina, Ciudad de México, México. ${ }^{4}$ Instituto Politécnico Nacional, Escuela Superior de Ingeniería Mecánica y Eléctrica-Unidad Zacatenco, Ciudad de México, México. ${ }^{5}$ Agencia de Evaluación de Tecnologías Sanitarias, Instituto de Salud Carlos III, Madrid, España, ${ }^{6}$ Instituto Nacional de Salud Pública, Centro de Investigación en Salud Poblacional, Cuernavaca, Morelos, México.
\end{abstract}

*Autor de correspondencia (israel.castillo@colpos.mx)

\section{RESUMEN}

Los aceites esenciales (AE) se han descrito como agentes bactericidas; sin embargo, estudios in vitro indican que algunos de sus constituyentes volátiles a concentraciones subletales pueden inhibir o promover los sistemas de percepción de quórum (PQ) en Pseudomonas aeruginosa. Es necesario explorar el papel que pueden tener las mezclas de volátiles sobre la virulencia y el establecimiento de la bacteria en sistemas in vivo. Este estudio se llevó a cabo para evaluar las propiedades bactericidas y antivirulencia de $A E$ obtenidos de Thymus vulgaris L., Cymbopogon citratus (DC.) Stapf y Tagetes lucida (Cav) contra $P$. aeruginosa. La actividad antivirulencia se evaluó mediante la inhibición de factores regulados por $\mathrm{PQ}$, como son la producción de piocianina y la actividad de proteasa y elastasa; además, se determinó la composición de volátiles y su capacidad para evitar el establecimiento de la infección en un modelo de lesión en ratones. Los ensayos in vitro mostraron que el $A E$ de $T$. vulgaris (AETV) únicamente exhibió actividad bactericida y el AE de $T$. lucida (AETL) redujo la producción de piocianina en $58 \%$ a concentración subletal de $200 \mu \mathrm{g} \mathrm{mL}^{-1}$. Para el caso del AE de $C$. citratus no se registró un efecto bactericida a la máxima concentración evaluada $(1000 \mu \mathrm{g} \mathrm{mL}-1)$, aunque redujo ligeramente la producción de piocianina (30\%). En los estudios in vivo únicamente el AETL y el Neosporin ${ }^{\circledR}$ (control positivo) interfirieron en el establecimiento de la bacteria, mientras que el AETV, contrario a lo esperado, lo favoreció, principalmente en zonas adyacentes a las lesiones. Se observó una correlación positiva entre el establecimiento de la bacteria y el número de constituyentes presentes en los AE. Estos resultados apoyan el uso de los AE evaluados para combatir $P$. aeruginosa y su posible aplicación en terapias antivirulencia.

Palabras clave: Piocianina, plantas aromáticas, modelo murino, imágenes termográficas, percepción de quórum.

\section{SUMMARY}

Essential oils (EO) have been described as bactericidal agents; however, in vitro studies indicate that some of their volatile constituents at sublethal concentrations may inhibit or promote quorum sensing (QS) systems in Pseudomonas aeruginosa. It is necessary to explore the role that volatile mixtures can play on virulence and in the establishment of bacteria on in vivo systems. The study was carried out to evaluate the bactericidal and antivirulence properties of EO obtained from Thymus vulgaris L., Cymbopogon citratus (DC.) Stapf and Tagetes lucida (Cav) against $P$. aeruginosa. Antivirulence activity was assessed by the inhibition of factors regulated by QS, such as pyocyanin production and protease and elastase activity; in addition the composition of volatiles and their ability to prevent the establishment of the infection in model of lesion in mice were determined. In vitro tests showed that $T$. vulgaris EO (TVEO) only exhibited bactericidal activity, and T. lucida EO (TLEO) reduced pyocyanin production by $58 \%$ at a sublethal concentration of $200 \mu \mathrm{g} \mathrm{mL}^{-1}$. In the case of the EO of $C$. citratus no bactericidal effect was registered at the maximum concentration evaluated $\left(1000 \mu \mathrm{g} \mathrm{mL}^{-1}\right)$, although it slightly reduced the pyocyanin production (30\%). In the in vivo studies only the TLEO and Neosporin ${ }^{\circledR}$ (positive control) interfered in the establishment of the bacteria, while the TVEO, contrary to the expected, favored the establishment of the bacterium, mainly in areas adjacent to the lesions. A positive correlation was observed between the establishment of the bacteria and the number of constituents present in the EO. These results support the use of the EO evaluated to combat $P$. aeruginosa and their possible application in anti-virulence therapies.

Index words: Pyocyanin, aromatic plants, murine model, thermographic imagery, quorum sensing.

\section{INTRODUCCIÓN}

Uno de los descubrimientos más importantes de la medicina fue el de los antibióticos, con los cuales se logró reducir la mortalidad causada por infecciones de origen bacteriano (Chastre, 2008). Actualmente, su efectividad se ha visto reducida por la constante aparición de cepas resistentes, así como por la propagación de los genes que la generan (Castillo-Juárez et al., 2017). La Organización Mundial de la Salud (OMS) ha advertido que, de continuar esta tendencia, en el año 2050 se podría entrar a una "era post-antibióticos", en la que las enfermedades infecciosas causarán más muertes que las ocasionadas por el cáncer y la diabetes mellitus tipo II (Aryee y Price, 2015). Dentro de la lista denominada "Patógenos Prioritarios Resistentes a Antibióticos" con prioridad crítica se encuentra Pseudomonas aeruginosa (Ganesh y Rai, 2016).

Esta bacteria es uno de los principales patógenos oportunistas que utilizan una gran variedad de factores 
de virulencia para establecerse y generar daño (Lee y Zhang, 2015). P. aeruginosa causa infecciones severas y persistentes en individuos inmunodeprimidos, como son pacientes con fibrosis quística, que reciben tratamientos de quimioterapia, y con síndrome de inmunodeficiencia adquirida (Driscoll et al., 2007). El patógeno presenta una alta capacidad intrínseca para desarrollar resistencia (Hancock y Speert, 2000; Montes-de-Oca-Márquez et al., 2017), además de que forma biopelículas y libera factores de virulencia como son elastasa, proteasa alcalina, proteasa LasA, hemolisina, rhamnolípidos y piocianina, entre otros (Lyczak et al., 2002).

Un nuevo enfoque a la idea convencional del uso de antibióticos es el desarrollo de terapias antivirulencia, con las cuales se busca inhibir los factores que generan el daño y evitar la aparición de resistencia (Mühlen y Dersch, 2015).

A la fecha se han identificado diversos blancos antivirulencia, como son la inhibición de sistemas de secreción bacteriana, los sistemas de dos componentes, enzimas clave en el daño al hospedero (Muñoz-Cazares et al., 2017) y la inhibición de la percepción de quórum (PQ), que hasta el momento es uno de los más estudiados debido a que se ha demostrado que tiene un papel central en la regulación de la expresión de virulencia global de las bacterias (Lee y Zhang, 2015). La PQ (comunicación bacteriana o quorum sensing) es un fenómeno que favorece el comportamiento multicelular en las bacterias, con el cual regulan de manera sincronizada en tiempo y espacio la expresión de factores de virulencia (MuñozCazares et al., 2018).

Los aceites esenciales (AE) obtenidos de especies vegetales han sido utilizados en la medicina tradicional de México y otras partes del mundo por poseer efectos antimicrobianos (Reichling, 2010); sin embargo, recientemente se ha descubierto que también son capaces de regular la virulencia (Packiavathy et al., 2012). Para el caso de $P$. aeruginosa se han identificado algunos componentes volátiles presentes en los AE que tienen la capacidad de inhibir o estimular la virulencia in vitro (Ahmad et al., 2015); esto parece indicar que la concentración y la clase de metabolitos secundarios presentes en los AE son un factor importante que determina el efecto bactericida, antivirulencia o promotor de la virulencia.

Thymus vulgaris L. y Cymbopogon citratus (DC.) Stapf son especies vegetales distribuidas en diversas partes del mundo con gran importancia comercial, principalmente por las propiedades bactericidas de sus AE (Negrelle y Gomes, 2007). Tagetes lucida Cav T. es una especie nativa de México que ha sido poco estudiada; se conoce como pericón, estragón mexicano, dragón dulce, yauhtli o caléndula mexicana; se usa tradicionalmente para curar trastornos gastrointestinales y mentales (Pérez-Ortega et al., 2016).

Hasta el momento no se tiene información sobre estudios in vivo que analicen el efecto de AE en infecciones causadas por $P$. aeruginosa. Aunado a esta situación, se ha observado que los sistemas de PQ que esta bacteria responden a estímulos externos como son la deficiencia de hierro, nutrientes y fosfato, así como moléculas del hospedero que pueden activarlo de manera independiente de los autoinductores o de la densidad bacteriana (Jensen et al., 2006; Lee y Zhang, 2015). Lo anterior sugiere que el efecto antivirulencia registrado para los volátiles de $\mathrm{AE}$ en las condiciones estandarizadas, como son los monocultivos in vitro, puede tener un resultado distinto en un sistema in vivo.

Por lo anterior, la presente investigación se centró en estudiar las propiedades antivirulencia (inhibidores de PQ) de los aceites esenciales obtenidos de T. vulgaris, C. citratus y $T$. lucida contra $P$. aeruginosa en estudios in vitro y su comparación en un modelo de lesión e infección en ratones.

\section{MATERIALES Y MÉTODOS}

\section{Material vegetal}

Plantas de T. vulgaris L. y C. citratus (DC.) Stapf se recolectaron en mayo y agosto de 2016, respetivamente, en el municipio de Ozumba, Estado de México, ubicado a $18^{\circ} 15^{\prime} 00^{\prime \prime}$ latitud norte y a $98^{\circ} 46^{\prime} 30^{\prime \prime}$ longitud oeste. El follaje se secó a $40{ }^{\circ} \mathrm{C}$ en una estufa de recirculación de aire durante 48 h. T. Iucida Cav. se recolectó en julio de 2016 en el predio la Siberia en Chapingo, Texcoco, Estado de México, ubicado a $19^{\circ} 26^{\prime} 56.1^{\prime \prime}$ latitud norte y a $98^{\circ}$ 48' 3.7" longitud oeste. El material vegetal limpio se secó bajo la sombra a temperatura ambiente y se almacenó en oscuridad a $4{ }^{\circ} \mathrm{C}$ hasta la extracción del AE. Los especímenes tipo se resguardaron en el Herbario-Hortorio del Colegio de Postgraduados, Campus Montecillo, Estado de México.

\section{Extracción de AE}

Los AE se obtuvieron mediante el método de hidrodestilación con ayuda de un aparato tipo Clevenger. El tiempo requerido para la extracción total fue de 3 h y se utilizaron $0.5 \mathrm{~kg}$ de hoja y tallos de T. vulgaris, $1 \mathrm{~kg}$ de hojas de $C$. citratus y $0.5 \mathrm{~kg}$ de inflorescencias de T. lucida. Los AE se secaron con sulfato de sodio anhidro, se transfirieron a un vial color ámbar de $1.5 \mathrm{~mL}$ con una pipeta Pasteur y se almacenaron hasta su análisis. 


\section{Análisis de cromatografía de gases/espectrometría de masas (CG/EM)}

La composición química de los $A E$ se determinó mediante el análisis de datos obtenidos por CG/EM. Un mg de muestra se disolvió en $50 \mu \mathrm{L}$ de diclorometano, de la cual se tomó $1 \mu \mathrm{L}$ y se inyectó al cromatógrafo de gases HP 6890 en modo Split (20:1). Los compuestos se separaron en una columna capilar HP5-ms $(30 \mathrm{~m} \times 0.25 \mathrm{~mm} \times 0.25$ $\mathrm{mm}$ ) mediante el siguiente programa de temperatura del horno del cromatógrafo: temperatura inicial de $40^{\circ} \mathrm{C}$ por 3 min, calentando hasta $300^{\circ} \mathrm{C}$ a una tasa de cambio de 10 ${ }^{\circ} \mathrm{C} \mathrm{min}^{-1}$, con un tiempo final de $2 \mathrm{~min}$. El gas portador fue He a una velocidad de flujo de $1 \mathrm{~mL} \mathrm{~min}^{-1}$. La temperatura del puerto de inyección fue de $250{ }^{\circ} \mathrm{C}$ y el retraso del disolvente fue de 3 min.

El cromatógrafo de gases se acopló a un detector selectivo de masas HP 5973 (Agilent Technologies, Canton, MA, USA). Las condiciones del espectrómetro fueron: energía de ionización electrónica de 70 eV, rango de escaneo de 30 a 550 uma, velocidad de lectura de 13.8 espectros $\mathrm{s}^{-1}$, temperatura de la cámara de ionización de $200{ }^{\circ} \mathrm{C}$ y temperatura de la línea de transferencia de $250{ }^{\circ} \mathrm{C}$. La adquisición y procesamiento de datos se realizaron con MassHunter Workstation software (Agilent Technologies, Canton, MA, USA). Los compuestos se identificaron con base en los patrones de fragmentación de los espectros de masas y los índices de Kovats calculados en relación con los tiempos de retención de una serie de alcanos $\left(\mathrm{C}_{7}-\mathrm{C}_{40}\right)$. Ambos se compararon con la información de los compuestos químicos en la base de datos del National Institute of Standards and Technology (NIST, 2019). Las cantidades relativas de los compuestos se expresaron como porcentajes del área de los picos en relación con el área total (\% área).

\section{Determinación de la actividad antivirulencia}

Para determinar la actividad antivirulencia se realizaron precultivos de $P$. aeruginosa PA14WT a partir de colonias individuales cultivadas en placas LB (Luria-Bertani, BD Difco ${ }^{\mathrm{TM}}$ ) a $37^{\circ} \mathrm{C}$ y se incubaron aeróbicamente por alrededor de 12 h en $5 \mathrm{~mL}$ de medio LB. Para cada ensayo se utilizó un cultivo fresco con una $\mathrm{OD}_{660 \mathrm{~nm}}=0.1\left(\sim 1 \times 10^{8} \mathrm{UFC} \mathrm{mL}^{-1}\right)$. Las concentraciones de AE diluido en dimetilsulfóxido (DMSO) fueron 40, 200 y $1000 \mu \mathrm{g} \mathrm{mL}-1$ finales. Como control negativo se usó DMSO y se realizaron tres repeticiones con cultivos independientes por duplicado. La concentración de DMSO en todos los ensayos fue $\leq 1 \%$. Después de $24 \mathrm{~h}$ de incubación se determinó el crecimiento $\left(\mathrm{DO}_{600 \mathrm{~nm}}\right)$ y 1.5 $\mathrm{mL}$ de cada cultivo se centrifugaron a 13,000 rpm por 2 min. Finalmente, los sobrenadantes se emplearon para determinar la producción de factores de virulencia.

\section{Producción de piocianina}

Se realizó una extracción con cloroformo, así como una extracción adicional con $\mathrm{HCl} 0.1 \mathrm{~N}$. Se midió la absorbancia del sobrenadante a $388 \mathrm{~nm}$ (O'Malley et al., 2004).

\section{Producción de proteasa}

Se determinó espectrofotométricamente mediante el ensayo de azul de remazol (Sigma) a $595 \mathrm{~nm}$. El medio de reacción contenía $20 \mathrm{mM}$ Tris- $\mathrm{HCl}$, pH 8.5 y $1 \mathrm{mM} \mathrm{CaCl}_{2}$ (Howe e Iglewski, 1984).

\section{Producción de elastasa}

Se realizó mediante el ensayo elastina rojo-Congo (Sigma) a $495 \mathrm{~nm}$, como se describió previamente por Ohman et al. (1980). El medio de reacción contenía Tris$\mathrm{HCl} 20 \mathrm{mM}, \mathrm{pH} 7.5$ y $\mathrm{CaCl}_{2} 1 \mathrm{mM}$.

\section{Modelo de lesión e infección con $P$. aeruginosa}

Se emplearon ratones CD1 machos de 4 a 5 semanas de edad adquiridos del Bioterio General de la Facultad de Estudios Superiores Iztacala-UNAM, los cuales se trasladaron y mantuvieron en un cuarto de experimentación del Colegio de Postgraduados-Campus Montecillo, México. En todo momento se siguieron las indicaciones de la NOM062-ZO0-1999 (SAGARPA, 2001) para manejo y uso de animales de laboratorio, así como las especificaciones del Reglamento para el Uso y Cuidado de Animales Destinados a la Investigación en el Colegio de Postgraduados. Se colocó un máximo de 15 animales por jaula $(32 \times 47 \times 20$ $\mathrm{cm}$ ) en camas de aserrín estéril, con alimentación estándar, con ciclos de luz-oscuridad de $12 \mathrm{~h}$ y una temperatura de alrededor de $27^{\circ} \mathrm{C}$.

\section{Preparación del inóculo bacteriano}

En $10 \mathrm{~mL}$ de medio LB se inocularon de dos a tres colonias de un cultivo de $P$. aeruginosa PA14 WT, y se incubó a $37{ }^{\circ} \mathrm{C}, 200 \mathrm{rpm}$ por 15 h; posteriormente, se ajustó a una $\mathrm{DO}_{660 \mathrm{~nm}}=0.06$, se centrifugó a 14,000 rpm por 1 min (Microfuge E Beckman, Brea, California, USA) y se realizaron dos lavados con PBS estéril. Para obtener el inoculo final, la pastilla bacteriana se homogenizó en $1 \mathrm{~mL}$ de PBS estéril. Para determinar el número de las UFC se utilizó el método de cuenta viable (Reynolds, 2005).

\section{Inducción de las lesiones e inoculación de $P$. aeruginosa}

Se desarrolló un modelo de infección basándose en el modelo murino de cicatrización de heridas (Dunn et al., 2013). Se emplearon de 5 a 6 animales por grupo, los 
cuales se anestesiaron con pentobarbital sódico (Pisa ${ }^{\circledR}$ Agropecuaria, Guadalajara, México) a una dosis de 45.8 $\mathrm{mg} \mathrm{kg}^{-1}$ de peso. Se eliminó el pelo de la parte dorsal con rasuradora eléctrica y navaja de afeitar; posteriormente, la zona se esterilizó con yodo y se les indujo dos lesiones por medio de una pinza sacabocados estéril Truper B17340. En las lesiones se colocaron con pegamento sintético (Kola loca ${ }^{\circledR}$ ) discos de plástico estériles de $1 \mathrm{~cm}$ con una abertura de $4 \mathrm{~mm}$ para evitar la contracción del musculo y mantener la lesión abierta (Dunn et al., 2013). Después, se inocularon $5 \mu \mathrm{L}\left(1 \times 10^{7}\right.$ UFC) en cada lesión y se dejó secar por 10 min. Finalmente, se administraron tópicamente los tratamientos que consistían en $5 \mu \mathrm{L}$ de AE o Neosporin ${ }^{\circledR}(500,000 \mathrm{U}$ de Polimixina B; 40,000 U de Bacitracina Zinc y $350 \mathrm{mg}$ de Neomicina) por cada $100 \mathrm{~g}$ en las lesiones del lado derecho, mientras que en el izquierdo se dejó sin tratamiento. Para evitar la infección de otros géneros bacterianos las lesiones se cubrieron con apósitos (tegaderm 3M) y con cinta adhesiva transparente (transpore $3 \mathrm{M}$ ) para restringir el acceso de los ratones a esta zona.

\section{Análisis de la infección en las lesiones}

A las 48 horas postinfección, los animales fueron anestesiados y se retiraron las cintas adhesivas junto con los apósitos. Debido a que P. aeruginosa produce pigmentos fluorescentes, como la piocianina, las lesiones se monitorearon con luz UV a $254 \mathrm{~nm}$ para observar la presencia de la bacteria.

\section{Análisis térmico de las lesiones}

Se empleó una cámara termográfica (FLIR T420 Thermal Imager, FLIR Systems, Boston, MA, USA) para determinar la temperatura del área de las lesiones. En un cuarto con temperatura controlada de 22 a $25^{\circ} \mathrm{C}$ se realizó la toma de las imágenes al momento en que los animales redujeron por completo su movimiento debido a la anestesia, con ayuda de un dispositivo diseñado para evitar la luz de fondo y mantener una distancia constante de $34 \mathrm{~cm}$ entre la lente de la cámara y el dorso del animal. El análisis de las imágenes se realizó con el software FLIR Tools disponible en http://www.flir.com/instruments/display/?id=54865 mediante el modo lava rango automático para análisis de las imágenes. Para todos los cálculos se empleó el cambio de temperatura promedio de la lesión sin tratamiento (o con tratamiento) versus la temperatura promedio de una zona sana (sin lesión).

La interpretación utilizada fue que un $\Delta_{\text {tpromedio }}$ cercano a cero es indicativo de que hubo menor establecimiento de la infección, por lo que el resultado del efecto de los AE en el establecimiento de $P$. aeruginosa en las lesiones puede ser: sin efecto $\left(\Delta_{\text {t promedio tratamiento }}=\Delta_{\text {t promedio control }}\right)$ favorece el establecimiento $\left(\Delta_{\text {t promedio tratamiento }}>\Delta_{\text {t promedio control }}\right)$ o interfiere en el establecimiento $\left(\Delta_{\text {t promedio tratamiento }}<\Delta_{\text {t promedio control }}\right)$

\section{Cuantificación de $P$. aeruginosa en las lesiones}

Discos de papel filtro estériles de $4 \mathrm{~mm}$ se colocaron en las lesiones por $10 \mathrm{~s}$ y después se colocaron en $0.5 \mathrm{~mL}$ de PBS estéril, donde se agitaron vigorosamente por $30 \mathrm{~s}$; posteriormente, se realizaron diluciones seriales 1/1000 hasta $1 \times 10^{10}$ y se sembraron $10 \mu \mathrm{L}$ de las diluciones pares. Las placas se dejaron incubar a $37^{\circ} \mathrm{C}$ por 24 h y se calculó el logaritmo del número de UFC por cada 4 mm de lesión.

\section{Análisis estadístico}

Los gráficos y los resultados fueron expresados en medias y desviaciones estándar, obtenidas por medio del programa Sigma-Plot 13. Las diferencias estadísticas significativas en ensayos in vitro fueron obtenidas por tres replicas independientes por duplicado y la significancia se verificó mediante análisis de varianza de un factor y pruebas de Bonferroni y $U$ de Mann-Whitney $(P<0.02)$. El análisis térmico se realizó mediante diagramas de caja y bigote y las diferencias significativas fueron calculadas mediante t de Student $(P<0.04)$ y $U$ de Mann-Whitney ( $P$ $<0.02$ ).

\section{RESULTADOS Y DISCUSIÓN}

\section{Composición química de los AE}

Se obtuvo un rendimiento de los $\mathrm{AE}$ de $0.22 \%$ en $T$. vulgaris (AETV), de $0.38 \%$ en C. citratus (AECC) y de $0.12 \%$ en T. Iucida (AETL). El análisis por CG/EM reveló diferencias entre la cantidad y composición, donde el AETV fue el más diverso con 15 compuestos, de los cuales el timol es el mayoritario con $43.28 \%$, seguido por el o-cimeno con 29.7 $\%$ y el isoterpinoleno con $7.2 \%$ (Cuadro 1). El AECC consta de 12 compuestos, de los cuales el geranial, con $47.66 \%$ y el neral, con 37.81 \% fueron los mayoritarios (Cuadro 2); de éstos, el timol y el neral presentan actividad antivirulencia contra P. aeruginosa al reducir la producción de piocianina in vitro (Ahmad et al., 2015).

El AETL fue el aceite esencial menos diverso con sólo 7 compuestos identificados, de los cuales el estragol fue el más abundante con $60.92 \%$, seguido por el metil eugenol con 19.58 \% (Cuadro 3). De estos compuestos no hay reporte hasta el momento sobre sus propiedades antivirulencia.

Los resultados obtenidos para los AETV y AECC concuerdan con los diversos estudios ya reportados en 
Cuadro 1. Composición química de los aceites esenciales encontrados en T. vulgaris.

\begin{tabular}{|c|c|c|c|c|c|c|c|}
\hline $\mathrm{No}^{+}$ & Compuesto & $\mathrm{FM}^{++}$ & $\mathrm{PM}^{9}$ & $\mid K^{9 \varphi}$ & IK lite & $\operatorname{TR}^{\mathrm{xx}}$ & $\% A^{f}$ \\
\hline 1 & Ciclofencheno & $\mathrm{C}_{10} \mathrm{H}_{16}$ & 136.1 & 934.7 & 927 & 7.92 & 2.49 \\
\hline 2 & Camfeno & $\mathrm{C}_{10} \mathrm{H}_{16}$ & 136.1 & 949.97 & 950 & 8.21 & 0.8 \\
\hline 3 & 2-Tuyeno & $\mathrm{C}_{10} \mathrm{H}_{16}$ & 136.1 & 978.3 & 978 & 8.75 & 0.47 \\
\hline 4 & b-Mirceno & $\mathrm{C}_{10} \mathrm{H}_{16}$ & 136.1 & 991.31 & 991 & 9.00 & 0.88 \\
\hline 5 & a-Terpineno & $\mathrm{C}_{10} \mathrm{H}_{16}$ & 136.1 & 1018.48 & 1018 & 9.48 & 0.68 \\
\hline 6 & o-Cimeno & $\mathrm{C}_{10} \mathrm{H}_{14}$ & 134.1 & 1028.34 & 1027 & 9.65 & 29.75 \\
\hline 7 & Isoterpinoleno & $\mathrm{C}_{10} \mathrm{H}_{16}$ & 136.1 & 1061.98 & 1057 & 10.22 & 7.25 \\
\hline 8 & Linalool & $\mathrm{C}_{10} \mathrm{H}_{18} \mathrm{O}$ & 154.1 & 1101.28 & 1101 & 10.90 & 5.92 \\
\hline 9 & Borneol & $\mathrm{C}_{10} \mathrm{H}_{18} \mathrm{O}$ & 154.1 & 1173.34 & 1173 & 12.03 & 0.83 \\
\hline 10 & 4-Terpineol & $\mathrm{C}_{10} \mathrm{H}_{18} \mathrm{O}$ & 154.1 & 1183.86 & 1175 & 12.19 & 0.98 \\
\hline 11 & a-Terpineol & $\mathrm{C}_{10} \mathrm{H}_{18} \mathrm{O}$ & 154.1 & 1196.94 & 1196 & 12.40 & 1.11 \\
\hline 12 & Timol & $\mathrm{C}_{10} \mathrm{H}_{14} \mathrm{O}$ & 150.1 & 1298.62 & 1298 & 13.87 & 43.28 \\
\hline 13 & Carvacrol & $\mathrm{C}_{10} \mathrm{H}_{14} \mathrm{O}$ & 150.1 & 1306.07 & 1307 & 13.98 & 3.22 \\
\hline 14 & b-cariofileno & $\mathrm{C}_{15} \mathrm{H}_{24}$ & 204.2 & 1434.74 & 1431 & 15.70 & 1.77 \\
\hline 15 & Oxido de b-cariofileno & $\mathrm{C}_{15} \mathrm{H}_{24} \mathrm{O}$ & 220.2 & 1602.97 & 1596 & 17.78 & 0.58 \\
\hline
\end{tabular}

†:Numeración asignada por orden de elución, ${ }^{\dagger+}:$ Fórmula molecular, ${ }^{\uparrow}:$ Peso molecular, ${ }^{\natural}:$ Índices de Kovats calculados con respecto a los datos de tiempo de retención obtenidos a partir de una columna capilar DB-5MS, x:Índices de Kovats reportados en literatura (NIST), xx:Tiempo de retención en $\min$, :\% de área relativa.

Cuadro 2. Composición química de los aceites esenciales encontrados en C. citratus.

\begin{tabular}{|c|c|c|c|c|c|c|c|}
\hline $\mathrm{No}^{+}$ & Compuesto & $\mathrm{FM}^{++}$ & $\mathrm{PM}^{9}$ & $I^{4}$ & IK lite & $\operatorname{TR}^{\mathrm{xx}}$ & $\% A^{f}$ \\
\hline 1 & Camfeno & $\mathrm{C}_{10} \mathrm{H}_{16}$ & 136.1 & 949.92 & 950 & 8.21 & 0.85 \\
\hline 2 & Metilheptenona & $\mathrm{C}_{8} \mathrm{H}_{14} \mathrm{O}$ & 126.1 & 987.31 & 987 & 8.92 & 0.75 \\
\hline 3 & 4-Nonanona & $\mathrm{C}_{9} \mathrm{H}_{18} \mathrm{O}$ & 142.1 & 1073.41 & 1030 & 10.42 & 1.11 \\
\hline 4 & Linalool & $\mathrm{C}_{10} \mathrm{H}_{18} \mathrm{O}$ & 154.1 & 1100.26 & 1100 & 10.88 & 0.98 \\
\hline 5 & Isoneral & $\mathrm{C}_{10} \mathrm{H}_{16} \mathrm{O}$ & 152.1 & 1165.94 & 1165 & 11.91 & 0.78 \\
\hline 6 & Isogeranial & $\mathrm{C}_{10} \mathrm{H}_{16} \mathrm{O}$ & 152.1 & 1184.44 & 1184 & 12.20 & 1.38 \\
\hline 7 & Neral & $\mathrm{C}_{10} \mathrm{H}_{16} \mathrm{O}$ & 152.1 & 1248.69 & 1249 & 13.15 & 37.81 \\
\hline 8 & Geraniol & $\mathrm{C}_{10} \mathrm{H}_{18} \mathrm{O}$ & 154.1 & 1259.03 & 1259 & 13.30 & 4.15 \\
\hline 9 & Geranial & $\mathrm{C}_{10} \mathrm{H}_{16} \mathrm{O}$ & 152.1 & 1278.34 & 1279 & 13.58 & 47.66 \\
\hline 10 & Acetato de geranilo & $\mathrm{C}_{12} \mathrm{H}_{20} \mathrm{O}_{2}$ & 196.1 & 1384.2 & 1384 & 15.04 & 3.11 \\
\hline 11 & Allo-Aromadendreno & $\mathrm{C}_{15} \mathrm{H}_{24}$ & 204.2 & 1434.82 & 1442 & 15.70 & 0.80 \\
\hline 12 & b-Himachaleno & $\mathrm{C}_{15} \mathrm{H}_{24}$ & 204.2 & 1528.73 & 1517 & 16.88 & 0.61 \\
\hline
\end{tabular}

${ }^{\mp}$ :Numeración asignada por orden de elución, ${ }^{+\dagger}:$ Fórmula molecular, ${ }^{4}:$ Peso molecular, ${ }^{4}:$ Indice de Kovats calculado con respecto a los datos de tiempo de retención obtenidos a partir de una columna capilar DB-5MS, x:Índices de Kovats reportados en literatura (NIST), xx:Tiempo de retención en $\min ,{ }^{f}: \%$ de área relativa. 
Cuadro 3. Composición química de los aceites esenciales encontrados en T. lucida.

\begin{tabular}{|c|c|c|c|c|c|c|c|}
\hline $\mathrm{No}^{+}$ & Compuesto & $\mathrm{FM}^{++}$ & $\mathrm{PM}^{4}$ & $1 K^{99}$ & IK lite & $\mathrm{TR}^{\mathrm{xx}}$ & $\% A^{f}$ \\
\hline 1 & Mirceno & $\mathrm{C}_{10} \mathrm{H}_{16}$ & 136.1 & 991.52 & 991 & 9.00 & 1.49 \\
\hline 2 & b-terpineno & $\mathrm{C}_{10} \mathrm{H}_{16}$ & 136.1 & 1050.09 & 1056 & 10.02 & 4.65 \\
\hline 3 & Linalool & $\mathrm{C}_{10} \mathrm{H}_{18} \mathrm{O}$ & 154.1 & 1100.89 & 1101 & 10.89 & 1.31 \\
\hline 4 & Estragol & $\mathrm{C}_{10} \mathrm{H}_{12} \mathrm{O}$ & 148.1 & 1207.31 & 1196 & 12.55 & 60.92 \\
\hline 5 & Metil eugenol & $\mathrm{C}_{11} \mathrm{H}_{14} \mathrm{O}_{2}$ & 178.1 & 1409.78 & 1410 & 15.38 & 19.58 \\
\hline 6 & Germacreno D & $\mathrm{C}_{15} \mathrm{H}_{24}$ & 204.2 & 1495.85 & 1496 & 16.48 & 0.64 \\
\hline 7 & Metil isoeugenol & $\mathrm{C}_{11} \mathrm{H}_{14} \mathrm{O}_{2}$ & 178.1 & 1504.72 & 1508 & 16.59 & 0.79 \\
\hline
\end{tabular}

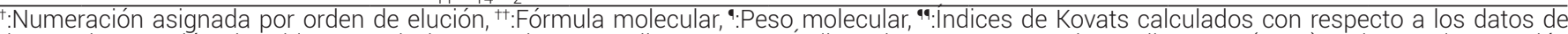
tiempo de retención obtenidos a partir de una columna capilar DB-5MS, x:Indices de Kovats reportados en literatura (NIST), xx:Tiempo de retención en $\min$, f:\% de área relativa.

cuanto a su composición química (Schmidt et al., 2012); de igual forma, está documentada su actividad bactericida para diversos microorganismos, así como para cepas de P. aeruginosa en estudios in vitro (Al-Bayati, 2008). Con respecto a los resultados obtenidos para $A E T L$, éstos representan el primer reporte sobre la identidad química de los componentes volátiles presentes en las inflorescencias de esta especie, así como de actividad bactericida y antivirulencia contra $P$. aeruginosa.

\section{Actividad antivirulencia de los AE contra $P$. aeruginosa}

Existe un elevado número de reportes que describen las actividades bactericidas de los AE y sus constituyentes individuales (Ahmad et al., 2015), pero la actividad antivirulencia sigue siendo poco estudiada. Para determinar las propiedades antivirulencia de los AE se evaluó su efecto en el crecimiento y sobre tres factores de virulencia regulados por sistemas de $P Q$ en $P$. aeruginosa (Castillo-Juárez et al., 2017).

EI AETV únicamente exhibió actividad bactericida a las concentraciones de 200 y $1000 \mu \mathrm{g} \mathrm{mL}^{-1}$ (Figura 1A); no obstante, aunque se observó una disminución en la producción de piocianina y la actividad de elastasa, se afectó el crecimiento. Para el caso de la actividad de proteasa no se observó este fenómeno, ya que no se redujo la actividad a pesar del fuerte efecto bactericida a $1000 \mu \mathrm{gL}^{-1}$ (Figura 1A). El AETL también mostró un efecto bactericida a la máxima concentración evaluada $\left(1000 \mu \mathrm{g} \mathrm{mL}^{-1}\right)$, pero exhibió una actividad antivirulencia a la concentración subletal de $200 \mu \mathrm{g} \mathrm{mL}^{-1}$, debido a que redujo la producción de piocianina en $58 \%$ sin afectar el crecimiento (Figura 1B). EI AECC no mostró actividad bactericida contra $P$. aeruginosa, pero sí un ligero efecto antivirulencia en la reducción de piocianina de 30 \% a 1000 $\mu \mathrm{gL}^{-1}$ (Figura 1B).
La actividad bactericida de los AE se debe a que dañan las membranas citoplasmáticas, la pared celular, las proteínas y disminuye la fuerza protón-motriz de la cadena respiratoria bacteriana (Friedman et al., 2002). Para el caso de la actividad antivirulencia aún se desconocen los mecanismos de acción o blancos específicos implicados (Ganesh y Rai, 2016; Muñoz-Cazares et al., 2018); no obstante, es claro que la concentración y el tipo de compuesto es determinante para que el AE exhiba un efecto bactericida o regulador de la virulencia in vitro.

\section{Eficacia de los AE para evitar el establecimiento de $P$. aeruginosa en lesiones}

Para evaluar la capacidad que tienen los AE para evitar el establecimiento de $P$. aeruginosa se diseñó un modelo de lesión e infección en ratones (Dunn et al., 2013). El establecimiento de la bacteria se determinó mediante análisis termográfico a las 48 h post-infección (Dini et al., 2015; Pérez-Bueno et al., 2015; Wilcox et al., 2009), así como por el método de cuenta viable. Como control positivo se administró en dosis única y de manera tópica una mezcla de compuestos bactericidas (Neosporin ${ }^{\circledR}$ ). Mediante el análisis térmico se registró una diferencia significativa entre las lesiones tratadas con Neosporin ${ }^{\circledR}$ y las no tratadas (Figura 2A, A1), así como una reducción en el número de UFC en el $80 \%$ de las lesiones tratadas (Figura 2, A2). Un efecto similar se registró en el análisis térmico con el AETL (Figura 2 C1), así como una reducción en las UFC en el $66.6 \%$ de las lesiones tratadas (Figura 2, C2).

Por otra parte y contrario a lo esperado, con el AETV, que mostró el mayor efecto bactericida en los ensayos in vitro, no se detectaron diferencias significativas en el análisis de las imágenes térmicas entre las lesiones tratadas y no tratadas (Figura 2B, B1); además, se registró un aumento en el número de UFC en el $83 \%$ de las lesiones en las que se administró el aceite (Figura 2, B2). Esta estimulación 

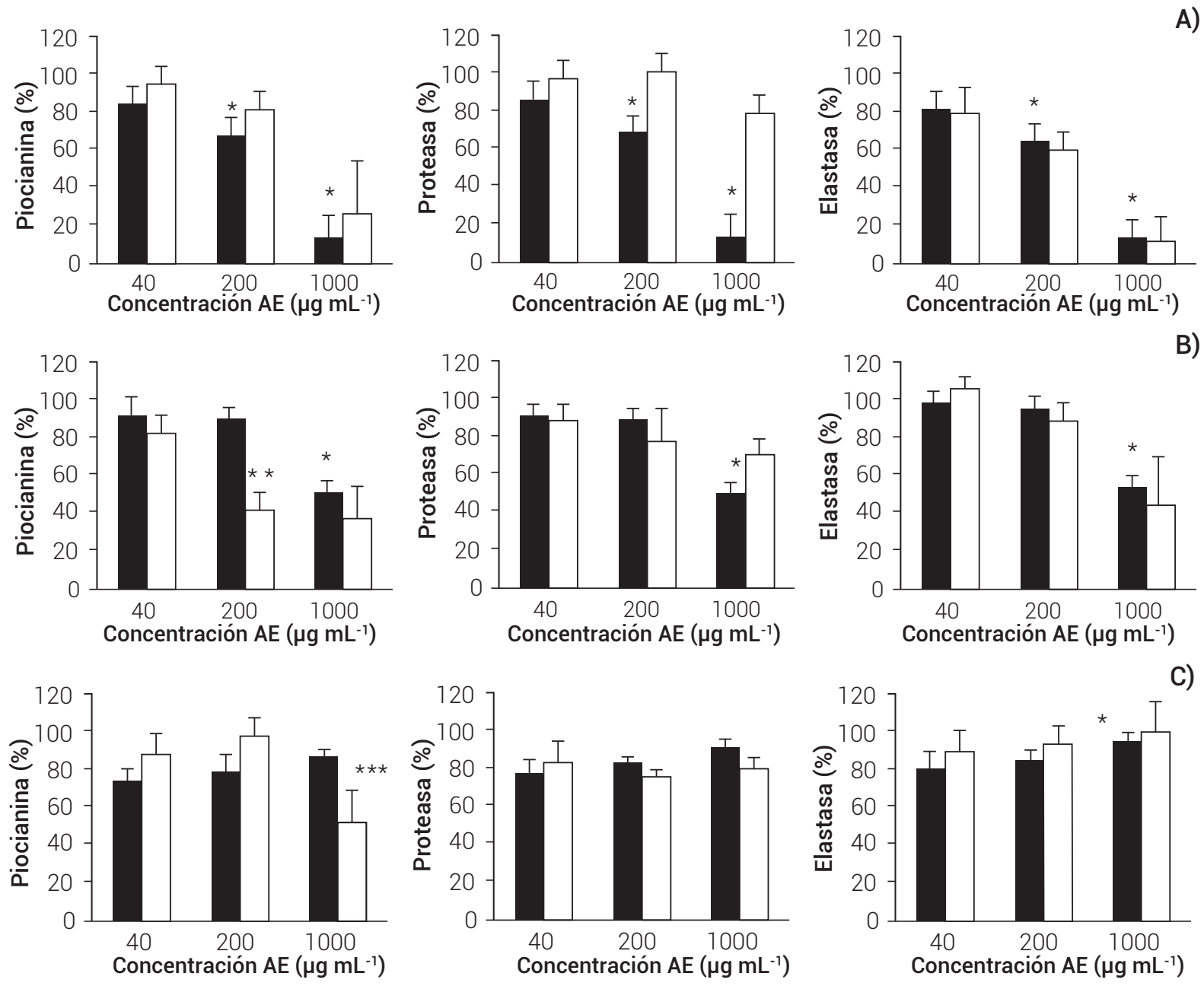

Figura 1. Actividad bactericida y antivirulencia de los aceite esenciales de T. vulgaris (A), T. lucida (B) y C. citratus (C) sobre $P$. aeruginosa. Columnas negras representan el porcentaje de crecimiento y blancas los distintos factores de virulencia evaluados. *: $\mathrm{P}<0.001, * *: \mathrm{P}<0.001$ ANOVA de un factor. Prueba de Bonferroni, ***:P $<0.02, \mathrm{U}$ de Mann-Whitney).

en el establecimiento se corroboró mediante un análisis visual con luz UV, con el cual es factible determinar la producción de pigmentos fluorescentes regulados por $\mathrm{PQ}$, como es la pioverdina. Se presentó una mayor fluorescencia en las lesiones tratadas con el AETV, incluso en zonas adyacentes donde se diseminó después de aplicarlo (Figura 3A); también, se observó el color verde característico de la infección por P. aeruginosa, debido a la combinación de la piocianina con la pioverdina (Figura 3B). Este es el primer reporte de una posible estimulación de virulencia bacteriana por AETV in vivo; sin embargo, hay que señalar que no queda claro el mecanismo mediante el cual ésto sucede. Son necesarios estudios encaminados a conocer el mecanismo exacto por el cual se presenta este fenómeno, en el que el grado de abrasión del AE, así como algunos factores del hospedero pueden ser determinantes para favorecer el establecimiento. Es posible que el AETV al aplicarse concentrado genere un daño al tejido y esto propicie un mejor ambiente para el establecimiento de $P$. aeruginosa. Nuevamente, son necesarios estudios adicionales a concentraciones menores, así como controles negativos en el que se analice su efecto sobre el tejido sin infección.

Este es el primer estudio que analiza el efecto de AE en el establecimiento de $P$. aeruginosa en un modelo murino. Dentro de los pocos estudios in vivo, destaca el mostrado por el AE de Murraya koenigii, el cual es capaz de atenuar los factores de virulencia de $P$. aeruginosa en un modelo de infección en el nematodo Caenorhabditis elegans (Husain et al., 2015). En el presente estudio también se registró un efecto positivo del AETL, el cual fue determinado por el análisis termográfico y corroborado por la presencia de la bacteria en las lesiones. Estos resultados señalan que es factible el uso de análisis termográficos como una herramienta que permite monitorear procesos infecciosos 

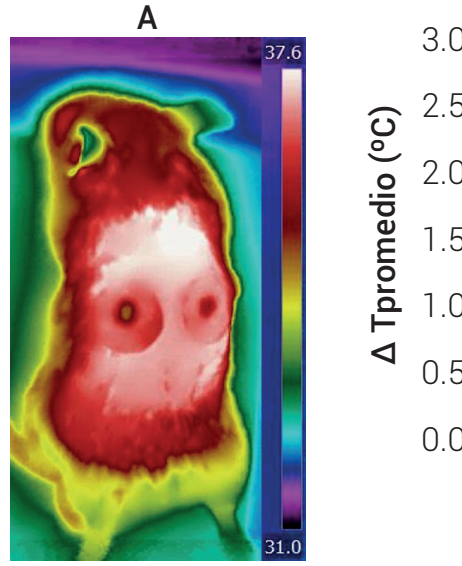

B
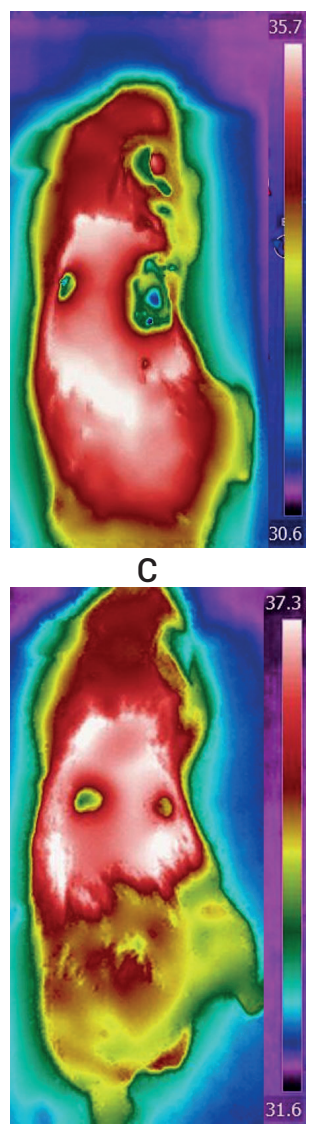

A1

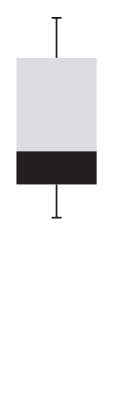

Control

Neosporin ${ }^{\circledR}$

B1

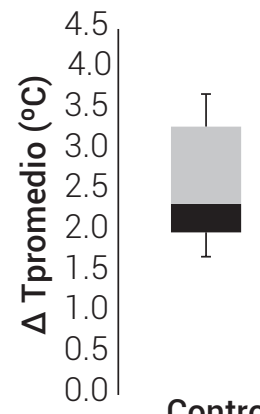

Control

T. vulgaris

$\mathrm{Cl}$

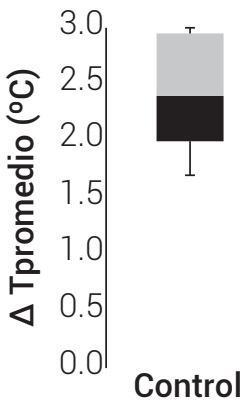

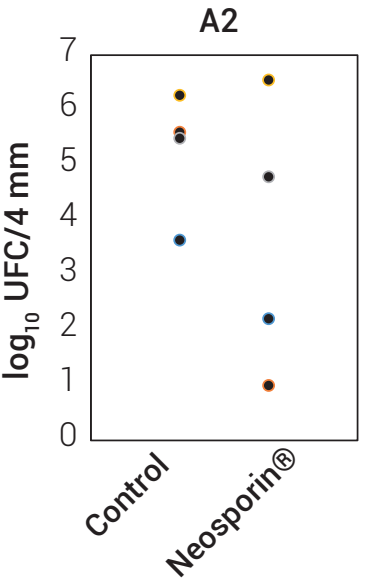
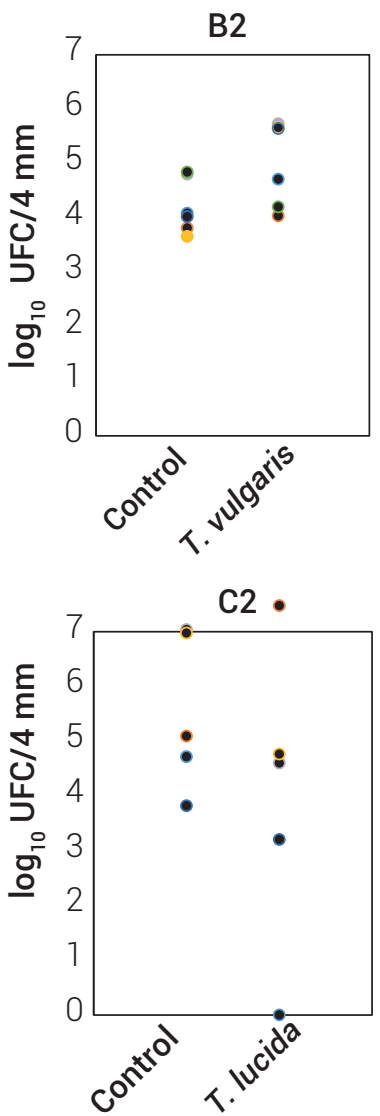

Figura 2. Efecto del Neosporin ${ }^{\circledR}$ y AE concentrados en el establecimiento de la infección de $P$. aeruginosa en un modelo de lesión en ratones. Imágenes térmicas en falso color representativas de las lesiones administradas tópicamente del lado derecho con Neosporin ${ }^{\circledR}(A)(n=5)$, T. vulgaris $(B)(n=6) \circ$ T. lucida $(C)(n=6)$ y del izquierdo sin tratamiento. $(A 1, B 1, C 1)$ Diagramas de caja y bigote obtenidas a partir de la diferencia de temperatura del área sin lesión y con lesiones inoculadas, con y sin tratamiento $\left(\Delta T=T_{\text {area sana }}-T_{\text {lesión con bacteria con y sin tratamiento }}\right)$ durante las primeras 48 h post infección. $(*$ : $P<0.04$ t de Student). (A2, B2, C2) Diagramas que muestran el número de UFC recuperadas de las lesiones que corresponden a un área de $4 \mathrm{~mm}$. 

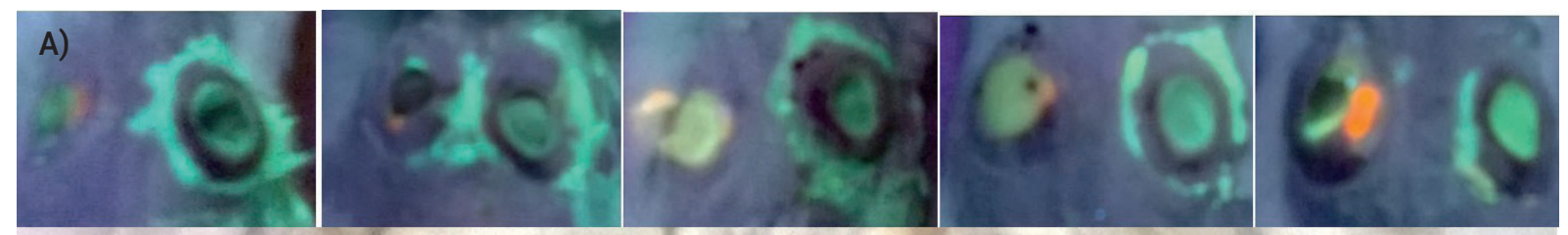

B)

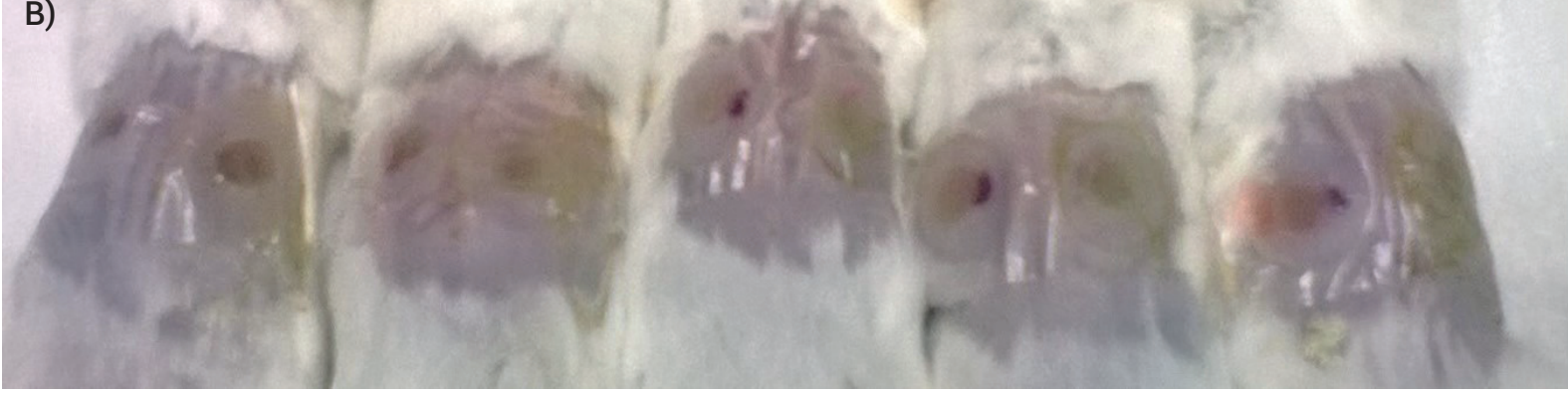

Figura 3. Imágenes de lesiones de un grupo experimental representativo tratadas con $T$. vulgaris (derecha) y sin tratamiento (izquierda) a las $48 \mathrm{~h}$ postinfección. A: luz UV a $254 \mathrm{~nm}$ y B: fotografía que muestra el dorso de los animales con el apósito, donde se observa una mayor producción de pigmentos en las lesiones tratadas con $T$. vulgaris.

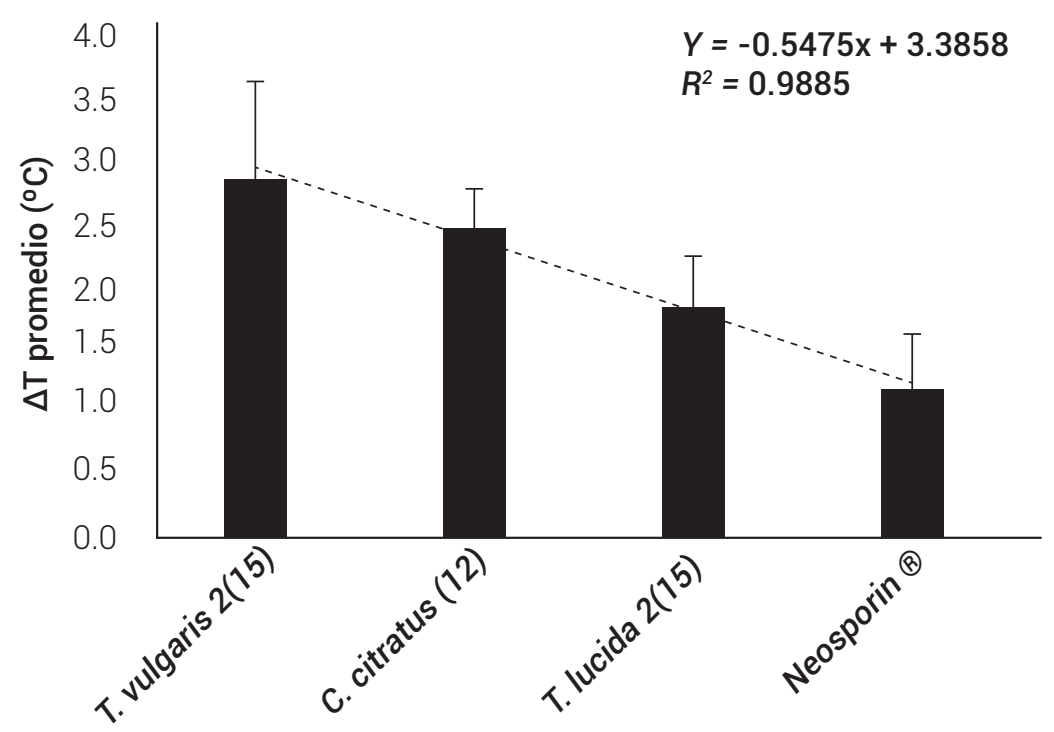

Figura 4. Relación entre la diversidad de compuestos volátiles presentes en los $\mathrm{AE}$ y su efecto en el establecimiento de $P$. aeruginosa en las lesiones. Neosporin ${ }^{\circledR}$ control positivo.

en lesiones, ya que se registró una tendencia similar de los distintos tratamientos entre los datos obtenidos por esta técnica y el conteo directo (Figura 2). En este respecto, algunos trabajos han señalado que es factible el uso de estas tecnologías en el ámbito médico y agronómico, con la ventaja de ser no invasivas, más rápidas, menos laboriosas y de menor costo (Dini et al., 2015; Pérez-Bueno et al., 2015; Wilcox et al., 2009).

Por otra parte, recientemente se identificó un grupo de volátiles que comúnmente están en los $A E$, con la propiedad de regular la producción de la piocianina in vitro (Ahmad et al., 2015). Es interesante que compuestos como (-)limoneno, (-)borneol y (-)carvona reducen la producción del pigmento, mientras que sus enantiómeros (+) la estimulan (Ahmad et al., 2015). En este trabajo, no se identificaron el limoneno ni la carvona en los $A E$; sin embargo, el borneol si se identificó en el AETV, pero no se determinó su quiralidad. Al igual que en el estudio in vitro, no se registró un aumento en los factores de virulencia analizados; no obstante, en este estudio se registró una asociación entre la diversidad de volátiles que 
presentan los AE y su actividad para estimular o reducir el establecimiento de $P$. aeruginosa (Figura 4). Este dato parece indicar que para $P$. aeruginosa un AE con un mayor grado de diversidad de constituyentes volátiles inducirá un efecto negativo in vivo.

\section{CONCLUSIONES}

Los AE analizados son distintos en cuanto a la diversidad y concentración de volátiles que contienen, lo que es determinante para que presenten actividad bactericida o reguladora de virulencia in vitro; sin embargo, su efecto para contrarrestar el establecimiento de $P$. aeruginosa in vivo es más complejo, incluso contraproducente si el AE es más diverso químicamente, como es el AETV; no obstante, el AETL tiene un potencial importante para ser empleado en el tratamiento de infecciones causadas por $P$. aeruginosa. El desarrollo de terapias antivirulencia con $\mathrm{AE}$ debe basarse en evaluaciones in vivo y con conocimiento de su composición química, ya que hacer inferencias directas de resultados obtenidos únicamente en ensayos in vitro puede resultar contraproducente.

\section{AGRADECIMIENTOS}

Este trabajo fue apoyado por donaciones de Proyectos de Desarrollo Científico para Resolver Problemas Nacionales/CONACyT México No. 2015-01-402. A B-H agradece a CONACYT su beca de Maestría en Ciencias. I C-J fue apoyado por el Fideicomiso-COLPOS 167304 y Cátedras-CONACyT proyecto 2112, R G-C por PAPIIT UNAM RA201116 y D P-CH por INFRA-2015 CONACyT No.256307.

\section{BIBLIOGRAFÍA}

Ahmad A., A. M. Viljoen and H. Y. Chenia (2015) The impact of plant volatiles on bacterial quorum sensing. Letters in Applied Microbiology 60:8-19, https://doi.org/10.1111/lam.12343

Al-Bayati F. A. (2008) Synergistic antibacterial activity between Thymus vulgaris and Pimpinella anisum essential oils and methanol extracts. Journal of Ethnopharmacology 116:403406, https://doi.org/10.1016/j.jep.2007.12.003

Aryee A. and N. Price (2015) Antimicrobial stewardship - can we afford to do without it? British Journal of Clinical Pharmacology 79:173181, https://doi.org/10.1111/bcp.12417

Castillo-Juárez I., L. E. López-Jácome, G. Soberón-Chávez, M. Tomás, J. Lee, P. Castañeda-Tamez, I. A. Hernández-Barragán, M. Y. CruzMuñiz, T. Maeda, T. K. Wood and R. García-Contreras (2017) Exploiting quorum sensing inhibition for the control of Pseudomonas aeruginosa and Acinetobacter baumannii biofilms. Current Topics in Medicinal Chemistry 17:19151927, https://doi.org/10.2174/1568026617666170105144104

Chastre J. (2008) Evolving problems with resistant pathogens. Clinical Microbiology and Infection 14:3-14, https://doi.org/10.1111/j.1469-0691.2008.01958.x

Dini V., P. Salvo, A. Janowska, F. Di Francesco, A. Barbini and M. Romanelli (2015) Correlation between wound temperature obtained with an infrared camera and clinical wound bed score in venous leg ulcers. Wounds 27:274-278.

Driscoll J. A., S. L. Brody and M. H. Kollef(2007) The epidemiology, pathogenesis and treatment of Pseudomonas aeruginosa infections. Drugs 67:351-368, https://doi.org/10.2165/00003495-200767030-00003

Dunn L., H. C. Prosser, J. T. Tan, L. Z. Vanags, M. K. Ng and C. A. Bursill (2013) Murine model of wound healing. Journal of Visualized Experiments 75:e50265, https://doi.org/10.3791/50265

Friedman M., P. R. Henika and R. E. Mandrell (2002) Bactericidal activities of plant essential oils and some of their isolated constituents against Campylobacter jejuni, Escherichia coli, Listeria monocytogenes and Salmonella enterica. Journal of Food Protection 65:15451560, https://doi.org/10.4315/0362-028X-65.10.1545

Ganesh P. S. and R. V. Rai (2016) Inhibition of quorum-sensing-controlled virulence factors of Pseudomonas aeruginosa by Murraya koenigii essential oil: a study in a Caenorhabditis elegans infectious model. Journal of Medical Microbiology 65:15281535, https://doi.org/10.1099/jmm.0.000385

Hancock R. E. W. and D. P. Speert (2000) Antibiotic resistance in Pseudomonas aeruginosa: mechanisms and impact on treatment. Drug Resistance Updates 3:247-255, https://doi.org/10.1054/drup.2000.0152

Howe T. R. and B. H. Iglewski (1984) Isolation and characterization of alkaline protease-deficient mutants of Pseudomonas aeruginosa in vitro and in a mouse eye model. Infection and Immunity 43:1058-1063.

Husain F. M., I. Ahmad, M. S. Khan, E. Ahmad, Q. Tahseen, M. S. Khan and N. A. Alshabib (2015) Sub-MICs of Mentha piperita essential oil and menthol inhibits AHL mediated quorum sensing and biofilm of Gram-negative bacteria. Frontiers in Microbiology 6:420, https://doi.org/10.3389/fmicb.2015.00420

Jensen V., D. Löns, C. Zaoui, F. Bredenbruch, A. Meissner, G. Dieterich, R. Münch and S. Häussler (2006) RhIR expression in Pseudomonas aeruginosa is modulated by the Pseudomonas quinolone signal via PhoBdependent and -independent pathways. Journal of Bacteriology 188:8601-8606, https://doi.org/10.1128/JB.01378-06

Lee J. and L. Zhang (2015) The hierarchy quorum sensing network in Pseudomonas aeruginosa. Protein \& Cell 6:26-41, https://doi. org/10.1007/s13238-014-0100-x

Lyczak J. B., C. L. Cannon and G. B. Pier (2002) Lung infections associated with cystic fibrosis. Clinical Microbiology Reviews 15:194-222, https://doi.org/10.1128/CMR.15.2.194-222.2002

Montes-de-Oca-Márquez C., C. T. Hernández-Delgado, J. Orozco-Martínez, A. M. García-Bores, J. G. Ávila-Acevedo, M. T. Ortiz-Melo, I. PeñalosaCastro, G. López-Moreno y R. Serrano-Parrales (2017) Actividad antibacteriana y antifúngica de Dalea carthagenensis (Jacq.) J. F. Macbr. Revista Fitotecnia Mexicana 40:161-168.

Mühlen S. and P. Dersch (2015) Anti-virulence strategies to target bacterial infections. In: How to Overcome the Antibiotic Crisis. Current Topics in Microbiology and Immunology, Vol. 398. M. Stadler and P. Dersch (eds.). Springer International Publishing. Cham, Switzerland. pp:147-183, https://doi.org/10.1007/82_2015_490

Muñoz-Cazares N., R. García-Contreras, M. Pérez-López and I. CastilloJuárez (2017) Phenolic compounds with anti-virulence properties. In: Phenolic Compounds. Biological Activity. M. Soto-Hernández, M. Palma-Tenango and M. R. GarcíaMateos (eds.). IntechOpen. London, UK. pp:139-167, https:// doi.org/10.5772/66367

Muñoz-Cazares N., R. García-Contreras, M. Soto-Hernández, M. MartínezVázquez and I. Castillo-Juárez (2018) Natural products with quorum quenching-independent antivirulence properties. In:Studies in Natural Products chemistry Vol. 57 T. I. Atta-ur-Rahman (ed.) Elsevier Science Publishers. Amsterdam, The Netherlands. pp:327351, https://doi.org/10.1016/B978-0-444-64057-4.00010-7

Negrelle R. R. B. and E. C. Gomes (2007) Cymbopogon citratus (DC.) Stapf: chemical composition and bilogical activities. Revista Brasileira de Plantas Medicinais 9:80-92.

NIST, National Institute of Standards and Technology (2019) Using the SMR catalog. National Institute of Standards and Technology. Gaithersburg, Maryland, USA. https://www.nist.gov/srm/ using-online-catalog (Julio 2019).

O'Malley Y. Q., K. J. Reszka, D. R. Spitz, G. M. Denning and B. E. Britigan (2004) Pseudomonas aeruginosa pyocyanin directly oxidizes glutathione and decreases its levels in airway epithelial cells. American Journal of Physiology-Lung Cellular and Molecular Physiology 287:L94-L103, https://doi.org/10.1152/ajplung.00025.2004

Ohman D. E., S. J. Cryz and B. H. Iglewski (1980) Isolation and characterization of Pseudomonas aeruginosa PAO mutant that produces altered 
elastase. Journal of Bacteriology 142:836-842

Packiavathy I. A. S. V., P. Agilandeswari, K. S. Musthafa, S. K. Pandian and A. V. Ravi (2012) Antibiofilm and quorum sensing inhibitory potential of Cuminum cyminum and its secondary metabolite methyl eugenol against Gram negative bacterial pathogens. Food Research International 45:85-92, https://doi.org/10.1016/j.foodres.2011.10.022

Pérez-Bueno M. L., M. Pineda, E. Díaz-Casado and M. Barón (2015) Spatial and temporal dynamics of primary and secondary metabolism in Phaseolus vulgarischallenged by Pseudomonas syringae. Physiologia Plantarum 153:161-174, https://doi.org/10.1111/ppl.12237

Pérez-Ortega G., M. E. González-Trujano, G. E. Ángeles-López, F. Brindis, H. Vibrans and R. Reyes-Chilpa (2016) Tagetes lucida Cav. ethnobotany, phytochemistry and pharmacology of its tranquilizing properties. Journal of Ethnopharmacology 181:221-228, https://doi.org/10.1016/j.jep.2016.01.040

Reichling J. (2010) Plant-microbe interactions and secondary metabolites with antibacterial, antifungal and antiviral properties. Annual Plant Reviews. 39:214-347, https://doi.org/10.1002/9781119312994.apr0420

Reynolds J. (2005) Serial dilution protocols. American Society for
Microbiology. Kent, Ohio, USA. https://www.asmscience.org/ docserver/fulltext/education/protocol/protocol.2884.pdf?expir es $=1564436863 \&$ id $=$ id\&accname $=$ guest $\&$ checksum $=627 F E B 8$ DF3DB9DC2284A0E697AF554F5 (Julio 2019).

SAGARPA, Secretaría de Agricultura, Ganadería, Desarrollo Rural, Pesca y Alimentación (2001) Norma Oficial Mexicana NOM-062 ZOO-1999. Especificaciones técnicas para la producción cuidado y uso de los animales de laboratorio. SAGARPA. Diario Oficial de la Federación. Primera Sección. 2 de agosto de 2001. México. D. F. pp:107-165

Schmidt E., J. Wanner, M. Hiiferl, L. Jirovetz, G. Buchbauer, V. Gochev, T. Girova, A. Stoyanova and M. Geissler (2012) Chemical composition olfactory analysis and antibacterial activity of Thymus vulgaris chemotypes geraniol, 4-thujanol/terpinen-4-ol, thymol and linalool cultivated in southern France. Natural Product Communications 7:1095-1098.

Wilcox C. S., J. Patterson and H. W. Cheng (2009) Use of thermography to screen for subclinical bumblefoot in poultry. Poultry Science 88:1176-1180, https://doi.org/10.3382/ps.2008-00446vvv 
